\title{
Evaluation of Non-Chemical Eco-Friendly Weed Management Approaches in HDPS Cotton
}

\author{
Kamble Anand Shankar ${ }^{1 *}$, A. S. Channabasavanna ${ }^{1}$, B. G. Koppalkar ${ }^{1}$, M. Y. \\ Ajayakumar $^{2}$, Mahadevswamy ${ }^{1}$ and A. Amaregouda ${ }^{1}$ \\ ${ }^{1}$ Department of Agronomy, ${ }^{2}$ AICRP on Cotton, College of Agriculture, University of \\ Agricultural Sciences, Raichur-584 104, Karnataka, India \\ *Corresponding author
}

\section{A B S T R A C T}

\begin{tabular}{l} 
Ke y w o r d s \\
$\begin{array}{l}\text { HDPS (High } \\
\text { density planting } \\
\text { system), Weed } \\
\text { density, Weed } \\
\text { index, Seed index } \\
\text { and Economics }\end{array}$ \\
\hline $\begin{array}{l}\text { Article Info } \\
\text { Accepted: } \\
\text { 24 October } 2020 \\
\text { Available Online: } \\
\text { 10 November } 2020\end{array}$ \\
\hline
\end{tabular}

Field experiment was conducted at Department of Agronomy, College of Agriculture, UAS, Raichur to study the "Evaluation of non-chemical eco-friendly weed management approaches in HDPS cotton" during the year of 2017-18 and 2018-19. The experiment was laid out in Randomized Completely Block Design with three replications. There were 14 treatments imposed viz., polythene mulch, paddy straw mulch, cotton stalk mulch, intercropping with green manures at 1:1 ratio (Sunnhemp and Cowpea), four different botanicals extracts@20\% as PE (Eucalyptus sp., Prosopis juliflora extract, Cassia tora and Parthenium hysterophorus), mechanical, cultural and their combination compared with weed free check, unweeded control and recommended practice. The two years pooled data results registered that, weed free check has recorded significantly greater seed cotton yield $\left(1372 \mathrm{~kg} \mathrm{ha}^{-1}\right)$ over rest of the treatments. It was followed by Cotton + Sunnhemp (1:1) subsequently in-situ mulching at 45 DAS (1299 kg ha $\left.{ }^{-1}\right)$, pendimethalin 38.7 CS @ $680 \mathrm{~g}$ a.i./ha as PE fb pyrithiobac sodium $10 \mathrm{EC} 75 \mathrm{~g}$ a.i./ha + quizolofop ethyl $37.5 \mathrm{~g}$ a.i./ha at 25 DAS as PoE $\left(1274 \mathrm{~kg} \mathrm{ha}^{-1}\right)$ and black polythene sheet mulch $\left(1262 \mathrm{~kg} \mathrm{ha}^{-1}\right)$. These treatments also recorded lower weed density at 50 DAS (4.9, 4.0 and 5.3 respectively), weed dry weight $\mathrm{m}^{-2}$ at 50 DAS $(5.5,4.9$ and 5.7 , respectively), weed control efficiency at 75 DAS (77.2, 81.2 and 75.3, respectively), weed index (4.60, 6.30 and 7.20, respectively), number of sympodials plant ${ }^{-2}$ at harvest (15.8, 17.7 and 15.2), number of bolls plant ${ }^{-2}$ at harvest $(16.4,16.7$ and 16.3 , respectively), net returns (Rs.52284, 49613 and $42825 \mathrm{ha}^{-1}$ ) and BC ratio (3.3, 3.0 and 2.4, respectively). The ecofriendly treatments viz., cotton + Sunnhemp (1:1) and in-situ mulching at 45 DAS, mulching with black polythene sheet, Cotton + Cowpea (1:1) and in-situ mulching at 45 DAS were at par with weed free check and they might be recommended as these were the best options.

\section{Introduction}

Cotton (Gossypium sp.) is popularly known as "the white gold" and also known as king of fibre crops grown under diverse agro-climatic conditions around the world. It provides fibre, a raw material for textile industry along with cotton seed and quality animal feed and 
biomass in the form of cotton stalks and plays a vital role in economy of the country. The high density planting system (HDPS) is now being conceived as an alternate production system having a potential for improving productivity and profitability, increasing efficiency, reducing input costs and minimizing risks associated with India's cotton production system. Of many problems faced by the cotton growers, the most troublesome one is the control of weeds particularly during early stages of crop growth. Venugopalan et al., (2009) reported a reduction in yield due to weeds in cotton crop to the extent of 50 to 85 per cent. Thus, if proper weed control measures are followed, there would be greater availability of nutrients and moisture for the benefit of crop (Jalis and Shah, 1982).

Optimum cotton yield and quality for high density planting cotton requires good weed control throughout the growing season. Cotton being a wide spaced and long duration crop suffers from heavy weed competition during the early stages of crop growth. Critical period of crop weed competition is 60 to 70 days from sowing, which may cause yield loss from 40 to 85 per cent depending upon the nature and intensity of weeds. Most often due to incessant rains during kharif season, hand weeding and inter cultivation (IC) become difficult in cotton. Further, labourer being scarce and costly, growers are forced depend on chemicals for weed control. While the continuous herbicidal applications can cause herbicide-resistant in weed stand has a negative impacts upon soil health, human health and environment. Natural products released from allelopathic and medicinal plant residues may help to reduce the use of synthetic herbicides for weed management.

Manual weed management practice is laborious and expensive. In spite of herbicides being effective in increasing yield, indiscriminate use of herbicides has resulted in serious ecological implications such as development of herbicide resistance weeds and shifts in weed population. Recently, research attention has been focused on to find out alternative strategies for chemical weed control in several crops. Reduction in herbicide use is one of major goals of modern agriculture and there is much emphasis on search for alternative weed management strategies that are cheap, safe and sustainable.

So, the extracts from the different plants which are having less residual effect compared to chemicals used to control the weeds. And farmers look for selective post emergence broad spectrum herbicide /herbicide mixtures. Extracts are considered as an effective, economical and environment friendly/ eco-friendly weed management approach. The slow initial growth coupled with indeterminate growth habit favours the growing of intercrops without affecting yield of cotton. Intercropping has unique capacity to raise the unit profitability without disturbing the cotton ecosystem.

Intercropping is the growing of two or more crops simultaneously in the alternative rows on the same piece of land in order to utilize available resources efficiently and obtaining more production per unit area (Lithourgidis et al., 2011). Two crops differing in rooting ability, nutrient requirements, height and canopy grow simultaneously with least competition (Lithourgidis et al., 2006). Weed density and biomass may substantially be reduced through intercropping (Poggio, 2005). Singh et al., (2003) indicated that growing companion plants, which are selectively allelopathic to weeds, may provide a cost effective alternative to the use of synthetic chemicals. Hence, the present investigation was carried out to study "ecofriendly weed management through non- 
chemical approaches in high density planting system of cotton".

\section{Materials and Methods}

Experiment was conducted for two consecutive years 2017-18 and 2018-19 at Department of Agronomy, College of Agriculture, UAS, Raichur. The experiment was laid out in Randomized Completely Block Design with three replications. Fourteen treatments comprised of $\mathrm{T}_{1}$ : Mulching with black polythene, $\mathrm{T}_{2}$ : Mulching with cotton stalk at $5 \mathrm{t} / \mathrm{ha}, \mathrm{T}_{3}$ : Mulching with paddy straw at $5 \mathrm{t} / \mathrm{ha}, \mathrm{T}_{4}$ : Cotton + Sunnhemp (1:1) and insitu mulching at 45 DAS, $\mathrm{T}_{5}$ : Cotton + Cowpea (1:1) and insitu mulching at 45 DAS.T 6 : Weeding with cycle weeder at 25 , 50 and 75 DAS.T. 7 : HW at 25 DAS and IC at 50 and 75 DAS. T 8 : Eucalyptus sp. extract @ $20 \%$ as PE fb IC at 50 and 75 DAS.T9: Prosopis juliflora extract @ 20\% as PE fb IC at 50 and 75 DAS.T 10 : Cassia tora @ 20\% as $\mathrm{PE}$ fb IC at 50 and 75 DAS.T $_{11}$ : Parthenium hysterophorus extract $20 \%$ as PE fb IC at 50 and 75 DAS, $\mathrm{T}_{12}$ : Pendimethalin $38.7 \mathrm{C} \mathrm{S}$ @ $680 \mathrm{~g}$ a.i./ha as PE fb Pyrithiobac Sodium 10EC $75 \mathrm{~g}$ a.i./ha + Quizolofop ethyl $37.5 \mathrm{~g}$ a.i./ha at 25 DAS as POE, $\mathrm{T}_{13}$ : Weed free check, $\mathrm{T}_{14}$ : Unweeded control. The recommended dose of fertilizer and spacing for cotton was 80:40:40 NPK kg/ha and 60 $\mathrm{cm} \times 20 \mathrm{~cm}$ respectively maintained for all the treatments. Fertilizer was applied in the form of urea, di ammonium phosphate and muriate of potash. Entire dose of phosphorus, 50 per cent of $\mathrm{N}$ and $\mathrm{K}$ were applied to cotton as basal placement by the side of seed line. The remaining 50 per cent of recommended dose of nitrogen and potassium was top dressed on 45 DAS by placement method. The fertilizers were placed $5 \mathrm{~cm}$ away from seed row and covered with soil. Treatments such as polythene sheet mulch, cotton stalk mulch and paddy straw mulch were mulched at the time of sowing, intercropping with sunnhemp and cowpea were grown and in situ mulched at 45 DAS and other herbicide chemicals are used as pre-emergence and post-emergence as per the treatment details. The fresh leaves of botanicals such as, Eucalyptus sp. Prosopis juliflora, Cassia tora and Parthenium hysterophorus cut into small species, soaked in alcohol and water@1:1 proportion and kept for overnight. After 12 hours, soaked leaves were ground with the help of mixer grinder. From the paste, the leaf extract of each botanical species was prepared by filtration which represented 100 per cent stock solution. From the stock solution, 20 per cent concentration was prepared and sprayed as per the treatment schedule. Observations on weed density, weed dry weight, weed control efficiency (WCE), weed Index (WI), plant height, bolls plant ${ }^{-1}$, boll weight, seed cotton yield and economics with respect to different treatments were recorded and analysed following the procedure given by Gomez and Gomez (1984) for RCBD. The data pertaining to weeds were transformed to square root scale of $\sqrt{ }(X+1)$ and analysed as suggested by Snedecor and Cochran (1967). Whenever significant difference existed, critical difference was constructed at 5 per cent probability level. That treatment, where the difference was not significant was denoted as NS.

\section{Results and Discussion}

The result registered that, at 25 DAS weed free check recorded significantly lower total weed density of $1.0 \mathrm{~m}^{-2}$ and was closely followed by application of pendimethalin 38.7 CS@680 g a.i./ha as PE $\left(3.3 \mathrm{~m}^{-2}\right)$. These treatments were significantly superior with each other and over other treatments. The highest weed density was recorded in unweeded control $\left(11.3 \mathrm{~m}^{-2}\right)$ and in weeding cycle weeder and hand weeding. At later stage such as 50 DAS and 75 DAS, application of pendimethalin 38.7 CS @ 680 
$\mathrm{g}$ a.i./ha as PE $f b$ pyrithiobac sodium $10 \mathrm{EC}$ $75 \mathrm{~g}$ a.i./ha + quizolofop ethyl $37.5 \mathrm{~g}$ a.i./ha at 25 DAS as POE $\left(4.0\right.$ and $3.6 \mathrm{~m}^{-2}$, respectively), mulching with black polythene sheet (5.2 and $4.8 \mathrm{~m}^{-2}$, respectively) and cotton + sunnhemp and in-situ mulching at 45 DAS (4.3 and $5.0 \mathrm{~m}^{-2}$, respectively) were on par with each other but significantly superior with unweeded check (Table 1). These treatments were followed by the application of different leaf extracts viz., Eucalyptus, Prosopis juliflora, Cassia tora and Parthenium hysterophorous @ 20\% as PE. The difference between leaf extractants was no significant but recorded fewer weeds over unweeded check. The lower weed density might be due to the prevention of biosysnthesis of branched chain amino acids, valine, leucine and isoleucine in chloroplast of susceptible weeds. And also intercropping with sunnhemp, cowpea and in-situ mulching at 45 DAS and black polythene sheet mulch reduces the weed population by suppressing the growth of weeds by intercepting the sunlight and has good tolerating ability to existing weed population. This could be attributed to lower utilization of resources by weeds like moisture, nutrient, light and space which resulted in lower weed density. Thus, effect of these treatments resulted in keeping weed density and dry weight below the critical level of competition. These results are in agreement with those reported by Shivashankar et al., (2017) and Satishkumar (2016).

The total weed dry weight was depicted the total weed density. The pooled data at 25 DAS recorded significantly lowest weed dry weight under weed free check $\left(1.0 \mathrm{~m}^{-2}\right)$ compared to unweeded control $\left(6.3 \mathrm{~g} \mathrm{~m}^{-2}\right)$, weeding with cycle weeder at 25 DAS $(6.1 \mathrm{~g}$ $\left.\mathrm{m}^{-2}\right)$ and $\mathrm{HW}$ at $25 \mathrm{DAS}\left(6.0 \mathrm{~g} \mathrm{~m}^{-2}\right)$. This was followed by the application of pendimethalin 38.7 CS @ $680 \mathrm{~g}$ a.i./ha as PE $\left(2.8 \mathrm{~g} \mathrm{~m}^{-2}\right)$ and mulching with black polythene $\left(3.4 \mathrm{~g} \mathrm{~m}^{-2}\right)$.
These treatments were on par with each other but significantly superior over other treatments. At later stages i.e. 50 DAS, unweeded control recorded significantly higher total dry weight of weeds $\left(11.3 \mathrm{~g} \mathrm{~m}^{-2}\right)$ as compared to other treatments. Significantly lower dry weight of weeds was recorded in weed free check $\left(1.0 \mathrm{~g} \mathrm{~m}^{-2}\right)$ and which was followed by application of pendimethalin 38.7 CS @ $680 \mathrm{~g}$ a.i./ha as PE fb pyrithiobac sodium 10EC $75 \mathrm{~g}$ a.i./ha + quizolofop ethyl $37.5 \mathrm{~g} \mathrm{a.i./ha}$ at $25 \mathrm{DAS}$ as POE $\left(4.4 \mathrm{~g} \mathrm{~m}^{-2}\right)$, cotton + sunnhemp (1:1) and in-situ mulching at 45 DAS $\left(5.1 \mathrm{~g} \mathrm{~m}^{-2}\right)$, mulching with black polythene sheet $\left(5.1 \mathrm{~g} \mathrm{~m}^{-2}\right)$, cotton + cowpea (1:1) and in-situ mulching at 45 DAS (5.3 g $\mathrm{m}^{-2}$ ) except mulching with cotton stalk, paddy straw and spray of leaf extracts @ 20\% as PE followed by IC at 50 and 75 DAS. Among the leaf extracts spray there was no significant difference was fond but was significantly superior over unweeded control (Table 1). Total weed weight was highest as weeding was not done in unweeded check. At 25 DAS, application of pendimethalin 38.7 CS@680 $\mathrm{g}$ a.i./ha as PE recorded on par total weed dry weight as an indication of control of weeds by herbicide and it was on par with black polythene sheet mulch as a result of no germination of weeds due to shade effect of black polythene sheet mulch. Significantly higher weed dry weight observed was due to higher weed density as they were directly proportional to each other. At later stages such as 50, 75, 100, 125, and at harvest application of pyrithiobac sodium and quizalofop ethyl as post-emergence have controlled later germinated weeds. Thus, integrated effect of pre and post emergence herbicides resulted in keeping weed density lower and thus showed lower dry weight of weeds Patel et al., (2013).

The data on weed index was indicated that, weed free check (0.0) registered lowest weed index and the higher weed index was recorded 
in unweeded check (37.8). Among different treatments, application pendimethalin $38.7 \mathrm{CS}$ @ $680 \mathrm{~g}$ a.i./ha as PE fb pyrithiobac sodium 10 EC $75 \mathrm{~g}$ a.i./ha + quizolofop ethyl $37.5 \mathrm{~g}$ a.i./ha at 25 DAS as POE (6.3) and agronomic practices viz., cotton + sunnhemp (1:1) and insitu mulching at 45 DAS (4.6) and mulching with black polythene sheet (7.2) was on par with weed free check and significantly superior over other treatments (Table 2). Among the different leaf extracts, Eucalyptus and Prosopis juliflora recorded significantly lower weed index over other two extracts (Cassia tora and Parthenium hyisterophorous). However these were significantly superior over unweeded control. The lower weed index was mainly due to effective weed control achieved and their by increased seed cotton yield. On contrary, higher weed index was as a result of poor control of weeds and thus reduced seed cotton yield. Similar results were also reported by Sarkar (2006).

The higher plant height was recorded with application of pendimethalin 38.7 CS @ 680 g a.i. ha ${ }^{-1}$ as PE $f b$ pyrithiobac sodium $10 \mathrm{EC}$ @ $75 \mathrm{~g}$ a.i. ha $\mathrm{a}^{-1}+$ quizolofop ethyl 5 EC @ $37.5 \mathrm{~g}$ a.i. ha ${ }^{-1}$ at $25 \mathrm{DAS}$ as PoE $(110.8 \mathrm{~cm})$, mulching with black polythene $(106.5 \mathrm{~cm})$, Sunnhemp $(107.8 \mathrm{~cm})$ and cowpea $(106 \mathrm{~cm})$ intercropping then in situ mulching at 45 DAS and HW at 25 DAS and IC at 50 and 75 DAS $(101.5 \mathrm{~cm})$ were on par with each other and superior over unweeded control and leaf extracts.

Significantly higher numbers of plant height, sympodials per plant, bolls per plant and boll weight were recorded in weed free check (11.0) over rest of the treatments but on par with the application of pendimethalin $38.7 \mathrm{CS}$ @ $680 \mathrm{~g}$ a.i. $\mathrm{ha}^{-1}$ as PE $f b$ pyrithiobac sodium 10 EC@ $75 \mathrm{~g}$ a.i. ha ${ }^{-1}+$ quizolofop ethyl 5 EC @ $37.5 \mathrm{~g}$ a.i. ha ${ }^{-1}$ at 25 DAS as PoE (10.3). black polythene mulch (9.2), cotton + sunnhemp (1:1) and in situ mulching at 45 DAS (9.3), cotton + cowpea (1:1) and in situ mulching at 45 DAS (9.1) and HW at 25 DAS at 50 and 75 DAS (9.0) produced higher number of sympodials per plant and were on par with chemical application and in turn were superior over unweeded control (Table 3). This was due to the enhanced plant height, dry matter production and nutrient uptake of the crop. This was also be due to long season weed control which was favourable for better growth and enhanced leaf area contributing for the activated photosynthesis and translocation of more photosynthates to sink which increased the boll weight (Nalini, 2010).

The pooled data over two years revealed that significantly greater seed cotton yield (1372 $\left.\mathrm{kg} \mathrm{ha}{ }^{-1}\right)$ in cotton + sunnhemp (1:1) subsequently in-situ mulching at 45 DAS (1299 $\left.\mathrm{kg} \mathrm{ha}^{-1}\right)$, pendimethalin $38.7 \mathrm{CS} @ 680$ $\mathrm{g}$ a.i./ha as $\mathrm{PE}$ fb pyrithiobac sodium $10 \mathrm{EC}$ $75 \mathrm{~g}$ a.i./ha + quizolofop ethyl $37.5 \mathrm{~g}$ a.i./ha at 25 DAS as POE (1274 kg ha $\left.{ }^{-1}\right)$ and black polythene sheet mulch $\left(1262 \mathrm{~kg} \mathrm{ha}^{-1)}\right.$ and were on par with weed free check. These treatments were on par with each other and superior over unweeded control (917 kg ha ${ }^{-1}$ ) (Table 4). Significantly higher yield, growth components in this treatment was mainly attributed to occurrence of less competition between cotton plants and weeds, leading to superior number of bolls and due to higher accumulation of photosynthates in leaves, stem and reproductive parts that resulted in better development of bolls and thereby increased yield. Thus, the crop under this treatment faced the least weed competition right from germination till the critical period. The lower number of sympodial branches per plant in unweeded control was due to severe weed competition on crop that has resulted in lower nutrient uptake and use of other growth resources and consequently stunted plant growth fewer number of fruiting branches. 
Table.1 Total weed density (no. $\mathrm{m}^{-2}$ ) at different growth stages in eco-friendly weed management through non-chemical approaches in HDPS cotton

\begin{tabular}{|c|c|c|c|c|c|c|c|c|c|c|c|c|}
\hline \multirow[t]{2}{*}{ Treatments } & \multicolumn{6}{|c|}{ Total weed density $\left(\right.$ no. $\left.\mathbf{~ m}^{-2}\right)$} & \multicolumn{6}{|c|}{ Weed dry weight $\left(\mathrm{g} \mathrm{m}^{-2}\right)$} \\
\hline & $\begin{array}{c}25 \\
\text { DAS }\end{array}$ & $\begin{array}{c}\mathbf{5 0} \\
\text { DAS }\end{array}$ & $\begin{array}{c}75 \\
\text { DAS }\end{array}$ & $\begin{array}{l}\text { 100 } \\
\text { DAS }\end{array}$ & $\begin{array}{l}125 \\
\text { DAS }\end{array}$ & $\begin{array}{c}\text { At } \\
\text { harvest }\end{array}$ & $\begin{array}{c}25 \\
\text { DAS }\end{array}$ & $\begin{array}{c}\mathbf{5 0} \\
\text { DAS }\end{array}$ & $\begin{array}{c}75 \\
\text { DAS }\end{array}$ & $\begin{array}{l}100 \\
\text { DAS }\end{array}$ & $\begin{array}{c}125 \\
\text { DAS }\end{array}$ & At harvest \\
\hline Mulching with black polythene & $\begin{array}{c}5.6 \\
(31.0)\end{array}$ & $\begin{array}{c}5.3 \\
(26.7)\end{array}$ & $\begin{array}{c}4.9 \\
(22.7)\end{array}$ & $\begin{array}{c}5.1 \\
(25.3)\end{array}$ & $\begin{array}{c}5.4 \\
(28.0)\end{array}$ & $\begin{array}{c}5.6 \\
(30.7)\end{array}$ & $3.4(10.8)$ & $\begin{array}{c}5.4 \\
(28.1) \\
\end{array}$ & $\begin{array}{c}5.7 \\
(31.1)\end{array}$ & $\begin{array}{c}6.0 \\
(35.4) \\
\end{array}$ & $\begin{array}{c}6.3 \\
(38.7)\end{array}$ & $\begin{array}{c}6.1 \\
(36.2)\end{array}$ \\
\hline Mulching with cotton stalk at $5 \mathrm{t} / \mathrm{ha}$ & $\begin{array}{c}7.3 \\
(53.7)\end{array}$ & $\begin{array}{c}7.6 \\
(56.3)\end{array}$ & $\begin{array}{c}6.9 \\
(47.3)\end{array}$ & $\begin{array}{c}7.6 \\
(58.0)\end{array}$ & $\begin{array}{c}7.1 \\
(49.7)\end{array}$ & $\begin{array}{c}7.4 \\
(53.7)\end{array}$ & $4.5(19.4)$ & $\begin{array}{c}8.3 \\
(69.1)\end{array}$ & $\begin{array}{c}8.0 \\
(63.7)\end{array}$ & $\begin{array}{c}9.1 \\
(81.4)\end{array}$ & $\begin{array}{c}9.7 \\
(93.2)\end{array}$ & $\begin{array}{l}7.9 \\
(61.2)\end{array}$ \\
\hline Mulching with paddy straw at $5 \mathrm{t} / \mathrm{ha}$ & $\begin{array}{c}7.1 \\
(49.3)\end{array}$ & $\begin{array}{c}7.5 \\
(54.7)\end{array}$ & $\begin{array}{c}6.2 \\
(37.3)\end{array}$ & $\begin{array}{c}7.0 \\
(48.0)\end{array}$ & $\begin{array}{c}6.8 \\
(45.0)\end{array}$ & $\begin{array}{c}6.7 \\
(44.4)\end{array}$ & $4.2(16.4)$ & $\begin{array}{c}7.4 \\
(54.4)\end{array}$ & $\begin{array}{c}6.9 \\
(47.4)\end{array}$ & $\begin{array}{c}9.3 \\
(84.9)\end{array}$ & $\begin{array}{c}9.5 \\
(90.3)\end{array}$ & $\begin{array}{l}7.7 \\
(57.8)\end{array}$ \\
\hline $\begin{array}{l}\text { Cotton + Sunnhemp }(1: 1) \text { and } \\
\text { mulching at } 45 \text { DAS. }\end{array}$ & $\begin{array}{c}6.0 \\
(35.3)\end{array}$ & $\begin{array}{c}4.9 \\
(23.3)\end{array}$ & $\begin{array}{c}4.3 \\
(17.3)\end{array}$ & $\begin{array}{c}5.0 \\
(23.7)\end{array}$ & $\begin{array}{c}4.7 \\
(21.3)\end{array}$ & $\begin{array}{c}5.0 \\
(24.3)\end{array}$ & $\begin{array}{c}3.9 \\
(14.6)\end{array}$ & $\begin{array}{c}5.1 \\
(25.1)\end{array}$ & $\begin{array}{c}5.5 \\
(28.8)\end{array}$ & $\begin{array}{c}5.8 \\
(32.2)\end{array}$ & $\begin{array}{c}6.2 \\
(37.6)\end{array}$ & $\begin{array}{c}5.8 \\
(32.3)\end{array}$ \\
\hline $\begin{array}{l}\text { Cotton + Cowpea (1:1) and mulching } \\
\text { at } 45 \text { DAS. }\end{array}$ & $\begin{array}{c}6.3 \\
(39.0)\end{array}$ & $\begin{array}{c}5.5 \\
(29.8)\end{array}$ & $\begin{array}{c}5.3 \\
(26.7)\end{array}$ & $\begin{array}{c}5.5 \\
(29.0)\end{array}$ & $\begin{array}{c}5.2 \\
(27.0)\end{array}$ & $\begin{array}{c}5.5 \\
(29.0)\end{array}$ & $4.3(17.2)$ & $\begin{array}{l}5.3 \\
(27.2)\end{array}$ & $\begin{array}{c}5.7 \\
(31.2)\end{array}$ & $\begin{array}{c}6.0 \\
(34.6)\end{array}$ & $\begin{array}{c}6.3 \\
(39.3)\end{array}$ & $\begin{array}{c}6.2 \\
(38.0)\end{array}$ \\
\hline $\begin{array}{l}\text { Weeding with cycle weeder at } 25,50 \\
\text { and } 75 \text { DAS. }\end{array}$ & $\begin{array}{c}11.5 \\
(131.0)\end{array}$ & $\begin{array}{c}5.9 \\
(34.0)\end{array}$ & $\begin{array}{c}5.8 \\
(33.0)\end{array}$ & $\begin{array}{c}6.0 \\
(35.3)\end{array}$ & $\begin{array}{c}6.9 \\
(46.7)\end{array}$ & $\begin{array}{c}7.1 \\
(50.0)\end{array}$ & $6.1(36.6)$ & $\begin{array}{c}5.6 \\
(30.1)\end{array}$ & $\begin{array}{c}6.1 \\
(36.5)\end{array}$ & $\begin{array}{c}6.2 \\
(38.0)\end{array}$ & $\begin{array}{c}6.6 \\
(42.7)\end{array}$ & $\begin{array}{l}7.3 \\
(51.7)\end{array}$ \\
\hline $\begin{array}{l}\text { HW at } 25 \text { DAS and IC at } 50 \text { and } 75 \\
\text { DAS. }\end{array}$ & $\begin{array}{c}11.5 \\
(130.7)\end{array}$ & $\begin{array}{c}5.7 \\
(31.7)\end{array}$ & $\begin{array}{c}5.6 \\
(30.7)\end{array}$ & $\begin{array}{c}6.2 \\
(37.3)\end{array}$ & $\begin{array}{c}6.6 \\
(43.3)\end{array}$ & $\begin{array}{c}7.0 \\
(48.0)\end{array}$ & $6.0(35.6)$ & $\begin{array}{c}5.6 \\
(30.1)\end{array}$ & $\begin{array}{c}5.9 \\
(34.1)\end{array}$ & $\begin{array}{c}6.1 \\
(36.6)\end{array}$ & $\begin{array}{c}6.5 \\
(40.9)\end{array}$ & $\begin{array}{l}7.2 \\
(51.0)\end{array}$ \\
\hline $\begin{array}{l}\text { Eucalyptus extract @ } 20 \% \text { as PE } f b \text { IC } \\
\text { at } 50 \text { and } 75 \text { DAS. }\end{array}$ & $\begin{array}{c}7.4 \\
(53.3)\end{array}$ & $\begin{array}{c}7.6 \\
(57.3)\end{array}$ & $\begin{array}{c}6.9 \\
(46.0\end{array}$ & $\begin{array}{c}7.4 \\
(53.7)\end{array}$ & $\begin{array}{c}7.0 \\
(49.7)\end{array}$ & $\begin{array}{c}7.2 \\
(51.3)\end{array}$ & $4.3(17.8)$ & $\begin{array}{l}8.3 \\
(68.7)\end{array}$ & $\begin{array}{c}8.8 \\
(76.2)\end{array}$ & $\begin{array}{c}9.0 \\
(79.4)\end{array}$ & $\begin{array}{c}9.5 \\
(89.1)\end{array}$ & $\begin{array}{c}7.5 \\
(54.9)\end{array}$ \\
\hline $\begin{array}{l}\text { Prosopis juliflora extract @ } 20 \% \text { as PE } \\
f b \text { IC at } 50 \text { and } 75 \text { DAS. }\end{array}$ & $\begin{array}{c}8.0 \\
(63.7)\end{array}$ & $\begin{array}{c}8.5 \\
(71.3)\end{array}$ & $\begin{array}{c}7.6 \\
(57.3)\end{array}$ & $\begin{array}{c}8.1 \\
(65.7)\end{array}$ & $\begin{array}{c}7.6 \\
(57.3)\end{array}$ & $\begin{array}{c}7.8 \\
(60.0)\end{array}$ & $4.7(21.4)$ & $\begin{array}{c}8.6 \\
(74.1)\end{array}$ & $\begin{array}{c}8.9 \\
(77.6)\end{array}$ & $\begin{array}{c}9.5 \\
(88.4)\end{array}$ & $\begin{array}{l}9.5 \\
(89.7)\end{array}$ & $\begin{array}{l}7.8 \\
(60.0)\end{array}$ \\
\hline $\begin{array}{l}\text { Cassia tora@ } 20 \% \text { as PE } f b \text { IC at } 50 \\
\text { and } 75 \text { DAS. }\end{array}$ & $\begin{array}{c}8.1 \\
(65.7)\end{array}$ & $\begin{array}{c}8.4 \\
(69.0)\end{array}$ & $\begin{array}{c}7.9 \\
(61.7)\end{array}$ & $\begin{array}{c}8.4 \\
(70.3) \\
\end{array}$ & $\begin{array}{c}7.9 \\
(61.7)\end{array}$ & $\begin{array}{c}8.0 \\
(63.0)\end{array}$ & $4.7(20.9)$ & $\begin{array}{c}8.8 \\
(75.8)\end{array}$ & $\begin{array}{c}9.0 \\
(80.0)\end{array}$ & $\begin{array}{c}9.5 \\
(89.5)\end{array}$ & $\begin{array}{c}9.7 \\
(92.5)\end{array}$ & $\begin{array}{c}7.9 \\
(62.5)\end{array}$ \\
\hline $\begin{array}{l}\text { Parthenium extract } 20 \% \text { as PE fb IC at } \\
50 \text { and } 75 \text { DAS. }\end{array}$ & $\begin{array}{c}8.3 \\
(68.7)\end{array}$ & $\begin{array}{c}8.8 \\
(77.0)\end{array}$ & $\begin{array}{c}8.3 \\
(67.3)\end{array}$ & $\begin{array}{c}8.7 \\
(74.0)\end{array}$ & $\begin{array}{c}8.4 \\
(69.7)\end{array}$ & $\begin{array}{c}8.3 \\
(68.7)\end{array}$ & $4.8(22.1)$ & $\begin{array}{c}9.1 \\
(82.2)\end{array}$ & $\begin{array}{c}9.2 \\
(83.9)\end{array}$ & $\begin{array}{c}9.7 \\
(92.3)\end{array}$ & $\begin{array}{l}9.8 \\
(94.2)\end{array}$ & $\begin{array}{c}8.1 \\
(64.3)\end{array}$ \\
\hline $\begin{array}{l}\text { Pendimethalin } 38.7 \text { C S @ } 680 \mathrm{~g} \text { a.i./ha } \\
\text { as PE } f b \text { Pyrithiobac Sodium } 10 \mathrm{EC} 75 \\
\text { g a.i./ha + Quizolofop ethyl } 37.5 \mathrm{~g} \\
\text { a.i./ha. at } 25 \text { DAS as PoE. }\end{array}$ & $\begin{array}{c}3.3 \\
(10.0)\end{array}$ & $\begin{array}{c}4.0 \\
(14.3)\end{array}$ & $\begin{array}{c}3.6 \\
(11.7)\end{array}$ & $\begin{array}{c}4.1 \\
(15.7)\end{array}$ & $\begin{array}{c}4.1 \\
(16.0)\end{array}$ & $\begin{array}{c}4.3 \\
(17.7)\end{array}$ & $2.8(6.8)$ & $\begin{array}{c}4.4 \\
(18.4)\end{array}$ & $\begin{array}{c}4.9 \\
(23.4)\end{array}$ & $\begin{array}{c}5.1 \\
(25.6)\end{array}$ & $\begin{array}{c}5.9 \\
(33.5)\end{array}$ & $\begin{array}{c}5.4 \\
(28.1)\end{array}$ \\
\hline Weed free check & $\begin{array}{c}1.0 \\
(0.0)\end{array}$ & $\begin{array}{c}1.0 \\
(0.0)\end{array}$ & $\begin{array}{c}1.0 \\
(0.0)\end{array}$ & $\begin{array}{c}1.0 \\
(0.0)\end{array}$ & $\begin{array}{c}1.0 \\
(0.0)\end{array}$ & $\begin{array}{c}1.0 \\
(0.0)\end{array}$ & $1.0(0.0)$ & $1.0(0.0)$ & $1.0(0.0)$ & $1.0(0.0)$ & $1.0(0.0)$ & $\begin{array}{l}1.0 \\
(0.0)\end{array}$ \\
\hline Unweeded control & $\begin{array}{c}11.8 \\
(137.7)\end{array}$ & $\begin{array}{c}12.3 \\
(149.3)\end{array}$ & $\begin{array}{c}11.2 \\
(124.0)\end{array}$ & $\begin{array}{c}11.2 \\
(125.0)\end{array}$ & $\begin{array}{c}11.2 \\
(125.7)\end{array}$ & $\begin{array}{c}11.5 \\
(132.0)\end{array}$ & $6.3(39.2)$ & $\begin{array}{c}11.3 \\
(125.9)\end{array}$ & $\begin{array}{c}11.4 \\
(128.3)\end{array}$ & $\begin{array}{c}11.2 \\
(125.3)\end{array}$ & $\begin{array}{c}12.3 \\
(150.4)\end{array}$ & $\begin{array}{c}10.5 \\
(109.2)\end{array}$ \\
\hline S.Em. \pm & 0.3 & 0.4 & 0.4 & 0.5 & 0.5 & 0.6 & 0.3 & 0.4 & 0.3 & 0.2 & 0.2 & 0.2 \\
\hline CD at $5 \%$ & 0.9 & 1.2 & 1.2 & 1.5 & 1.3 & 1.9 & 0.8 & 1.2 & 0.9 & 0.7 & 0.6 & 0.7 \\
\hline
\end{tabular}

* Figures in parenthesis indicate original values $* *$ Values are square root transformed $\sqrt{ } \mathrm{x}+1$ 
Table.2 Weed control efficiency (\%) at different growth stages in eco-friendly weed management through non-chemical approaches in HDPS cotton

\begin{tabular}{|c|c|c|c|c|c|c|}
\hline \multirow[t]{2}{*}{ Treatments } & \multicolumn{6}{|c|}{ WCE $(\%)$} \\
\hline & $\begin{array}{c}25 \\
\text { DAS }\end{array}$ & $\begin{array}{c}\mathbf{5 0} \\
\text { DAS }\end{array}$ & $\begin{array}{c}75 \\
\text { DAS }\end{array}$ & $\begin{array}{l}\text { 100 } \\
\text { DAS }\end{array}$ & $\begin{array}{c}125 \\
\text { DAS }\end{array}$ & $\begin{array}{c}\text { At } \\
\text { harvest }\end{array}$ \\
\hline Mulching with black polythene & 70.2 & 77.5 & 75.3 & 71.6 & 73.5 & 67.3 \\
\hline Mulching with cotton stalk at $5 \mathrm{t} / \mathrm{ha}$ & 50.4 & 45.3 & 49.9 & 35.0 & 35.9 & 43.8 \\
\hline Mulching with paddy straw at $5 \mathrm{t} / \mathrm{ha}$ & 56.7 & 56.8 & 63.8 & 32.1 & 38.1 & 47.0 \\
\hline Cotton + Sunnhemp (1:1) and mulching at 45 DAS. & 61.3 & 79.8 & 77.2 & 74.3 & 74.3 & 70.6 \\
\hline Cotton + Cowpea (1:1) and mulching at 45 DAS. & 54.0 & 78.2 & 75.2 & 72.3 & 73.1 & 66.0 \\
\hline Weeding with cycle weeder at 25,50 and 75 DAS. & 2.9 & 75.9 & 71.2 & 69.6 & 70.5 & 51.7 \\
\hline HW at 25 DAS and IC at 50 and 75 DAS. & 5.9 & 75.8 & 72.7 & 70.5 & 71.9 & 51.9 \\
\hline Eucalyptus extract @ $20 \%$ as PE $f b$ IC at 50 and 75 DAS. & 51.7 & 45.3 & 40.4 & 36.4 & 39.2 & 48.8 \\
\hline $\begin{array}{l}\text { Prosopis juliflora extract @ } 20 \% \text { as PE } f b \text { IC at } 50 \text { and } 75 \\
\text { DAS. }\end{array}$ & 43.7 & 41.0 & 38.3 & 29.2 & 38.7 & 44.3 \\
\hline Cassia tora@ 20\% as PE $f b$ IC at 50 and 75 DAS. & 45.0 & 39.6 & 36.5 & 28.4 & 36.9 & 42.6 \\
\hline Parthenium extract $20 \%$ as PE fb IC at 50 and 75 DAS. & 41.4 & 34.7 & 33.9 & 26.0 & 35.8 & 40.9 \\
\hline $\begin{array}{l}\text { Pendimethalin } 38.7 \text { C S @ } 680 \text { g a.i./ha as PE } f b \text { Pyrithiobac } \\
\text { Sodium } 10 E C 75 \text { g a.i./ha + Quizolofop ethyl } 37.5 \text { g a.i./ha. at } \\
25 \text { DAS as PoE. }\end{array}$ & 82.0 & 85.3 & 81.2 & 79.4 & 77.2 & 74.3 \\
\hline Weed free check & 100.0 & 100.0 & 100.0 & 100.0 & 100.0 & 100.0 \\
\hline Unweeded control & 0.0 & 0.0 & 0.0 & 0.0 & 0.0 & 0.0 \\
\hline S.Em. \pm & 7.2 & 5.4 & 3.4 & 3.0 & 2.6 & 2.6 \\
\hline CD at $5 \%$ & 20.9 & 15.7 & 9.9 & 8.8 & 7.5 & 7.6 \\
\hline
\end{tabular}


Table.3 Growth and yield parameters of cotton at harvest in eco-friendly weed management through non-chemical approaches in HDPS cotton

\begin{tabular}{|c|c|c|c|c|}
\hline Treatments & $\begin{array}{l}\text { Plant height } \\
\text { (cm) }\end{array}$ & $\begin{array}{c}\text { Sympodials per } \\
\text { plant }\end{array}$ & $\begin{array}{c}\text { No, of Bolls per } \\
\text { plant }\end{array}$ & $\begin{array}{c}\text { Boll weight } \\
\text { (g) }\end{array}$ \\
\hline Mulching with black polythene & 106.5 & 15.2 & 16.3 & 2.77 \\
\hline Mulching with cotton stalk at $5 \mathrm{t} / \mathrm{ha}$ & 97.7 & 14.2 & 14.1 & 2.48 \\
\hline Mulching with paddy straw at $5 \mathrm{t} / \mathrm{ha}$ & 100.7 & 14.6 & 14.7 & 2.52 \\
\hline Cotton + Sunnhemp (1:1) and mulching at 45 DAS. & 107.8 & 15.8 & 16.4 & 2.87 \\
\hline Cotton + Cowpea (1:1) and mulching at 45 DAS. & 106.3 & 15.5 & 16.1 & 2.78 \\
\hline Weeding with cycle weeder at 25,50 and 75 DAS. & 99.3 & 14.7 & 15.2 & 2.45 \\
\hline HW at 25 DAS and IC at 50 and 75 DAS. & 101.5 & 15.4 & 16.0 & 2.50 \\
\hline Eucalyptus extract @ $20 \%$ as PE $f b$ IC at 50 and 75 DAS. & 96.2 & 14.5 & 14.4 & 2.45 \\
\hline Prosopis juliflora extract @ 20\% as PE $f b$ IC at 50 and 75 DAS. & 94.5 & 14.2 & 13.3 & 2.42 \\
\hline Cassia tora@ 20\% as PE $f b$ IC at 50 and 75 DAS. & 94.4 & 14.0 & 13.3 & 2.43 \\
\hline Parthenium extract $20 \%$ as PE fb IC at 50 and 75 DAS. & 93.4 & 14.0 & 13.3 & 2.41 \\
\hline $\begin{array}{l}\text { Pendimethalin } 38.7 \text { C S @ } 680 \text { g a.i./ha as PE } f b \text { Pyrithiobac } \\
\text { Sodium 10EC } 75 \text { g a.i./ha + Quizolofop ethyl } 37.5 \text { g a.i./ha. at } \\
25 \text { DAS as PoE. }\end{array}$ & 110.8 & 17.7 & 16.7 & 2.97 \\
\hline Weed free check & 115.8 & 18.8 & 18.3 & 3.32 \\
\hline Unweeded control & 86.1 & 11.2 & 10.7 & 2.13 \\
\hline S.Em. \pm & 1.6 & 1.0 & 0.40 & 0.1 \\
\hline CD at $5 \%$ & 4.8 & 2.9 & 1.21 & 0.2 \\
\hline
\end{tabular}


Table.4 Yield and economics in eco-friendly weed management through non-chemical approaches in HDPS cotton

\begin{tabular}{|c|c|c|c|c|c|c|c|c|c|c|c|c|}
\hline \multirow[t]{2}{*}{ Treatments } & \multicolumn{3}{|c|}{ Yield (kg/ha) } & \multicolumn{3}{|c|}{ Gross returns (Rs/ha) } & \multicolumn{3}{|c|}{ Net Returns (Rs/ha) } & \multicolumn{3}{|c|}{ B:C ratio } \\
\hline & 2017 & 2018 & Pooled & 2017 & 2018 & Pooled & 2017 & 2018 & Pooled & 2017 & 2018 & Pooled \\
\hline Mulching with black polythene & 1293 & 1230 & 1262 & 77596 & 69383 & 73490 & 46827 & 38822 & 42825 & 2.5 & 2.3 & 2.4 \\
\hline Mulching with cotton stalk at $5 \mathrm{t} / \mathrm{ha}$ & 1072 & 1063 & 1068 & 64322 & 58724 & 61523 & 39653 & 35773 & 37713 & 2.6 & 2.6 & 2.6 \\
\hline Mulching with paddy straw at $5 \mathrm{t} / \mathrm{ha}$ & 1246 & 1174 & 1210 & 74744 & 66531 & 70638 & 49025 & 40783 & 44904 & 2.9 & 2.7 & 2.8 \\
\hline $\begin{array}{l}\text { Cotton + Sunnhemp }(1: 1) \text { and } \\
\text { mulching at } 45 \text { DAS. }\end{array}$ & 1340 & 1258 & 1299 & 80370 & 71427 & 75899 & 56901 & 47667 & 52284 & 3.4 & 3.2 & 3.3 \\
\hline $\begin{array}{l}\text { Cotton + Cowpea (1:1) and mulching } \\
\text { at } 45 \text { DAS. }\end{array}$ & 1225 & 1182 & 1204 & 73526 & 66210 & 69868 & 50557 & 44008 & 47283 & 3.2 & 3.1 & 3.1 \\
\hline $\begin{array}{l}\text { Weeding with cycle weeder at } 25,50 \\
\text { and } 75 \text { DAS. }\end{array}$ & 980 & 993 & 986 & 58826 & 54256 & 56541 & 32757 & 30475 & 31616 & 2.3 & 2.3 & 2.3 \\
\hline $\begin{array}{l}\text { HW at } 25 \text { DAS and IC at } 50 \text { and } 75 \\
\text { DAS. }\end{array}$ & 1069 & 1155 & 1112 & 64115 & 61148 & 62632 & 35646 & 37011 & 36328 & 2.3 & 2.4 & 2.3 \\
\hline $\begin{array}{l}\text { Eucalyptus extract @ } 20 \% \text { as PE } f b \\
\text { IC at } 50 \text { and } 75 \text { DAS. }\end{array}$ & 1173 & 1257 & 1215 & 70363 & 65391 & 68014 & 44294 & 41277 & 42922 & 2.7 & 2.7 & 2.7 \\
\hline $\begin{array}{l}\text { Prosopis juliflora extract @ } 20 \% \text { as } \\
\text { PE } f b \text { IC at } 50 \text { and } 75 \text { DAS. }\end{array}$ & 1121 & 1144 & 1133 & 67252 & 63715 & 65848 & 41183 & 39601 & 40756 & 2.6 & 2.6 & 2.6 \\
\hline $\begin{array}{l}\text { Cassia tora @ } 20 \% \text { as PE } f b \text { IC at } 50 \\
\text { and } 75 \text { DAS. }\end{array}$ & 1148 & 1093 & 1120 & 68859 & 61612 & 65236 & 42790 & 37498 & 40144 & 2.6 & 2.6 & 2.6 \\
\hline $\begin{array}{l}\text { Parthenium extract } 20 \% \text { as PE fb IC } \\
\text { at } 50 \text { and } 75 \text { DAS. }\end{array}$ & 1145 & 1114 & 1130 & 68730 & 62123 & 65426 & 42661 & 38009 & 40334 & 2.6 & 2.6 & 2.6 \\
\hline $\begin{array}{l}\text { Pendimethalin } 38.7 \text { C S @ } 680 \mathrm{~g} \\
\text { a.i./ha as PE } f b \text { Pyrithiobac Sodium } \\
\text { 10EC } 75 \text { g a.i./ha + Quizolofop ethyl } \\
\text { 37.5 g a.i./ha. at } 25 \text { DAS as PoE. }\end{array}$ & 1308 & 1239 & 1274 & 78508 & 70060 & 74284 & 52859 & 46366 & 49613 & 3.1 & 2.9 & 3.0 \\
\hline Weed free check & 1467 & 1277 & 1372 & 87993 & 75455 & 81724 & 51124 & 35337 & 43230 & 2.4 & 2.0 & 2.2 \\
\hline Unweeded control & 906 & 927 & 917 & 54367 & 50418 & 52393 & 31898 & 30487 & 31192 & 2.4 & 2.5 & 2.5 \\
\hline S.Em. \pm & 55 & 62 & 47 & 3317 & 3400 & 2691 & 3317 & 3400 & 2691 & 0.1 & 0.1 & 0.1 \\
\hline CD at $5 \%$ & 161 & 180 & 137 & 9642 & 9885 & 7823 & 9642 & 9885 & 7823 & 0.4 & 0.4 & 0.3 \\
\hline
\end{tabular}


The results obtained were in consonance with the study conducted by Sathishkumar (2016) and Madavi (2016). More boll weight in this treatment due to the enhanced plant height, dry matter production and nutrient uptake of the crop. This was also be due to the season long weed control which was favourable for better growth and enhanced leaf area contributed for the activated photosynthesis and translocation of more photosynthates to sink which increased the boll weight (Nalini, 2010). Higher growth parameter mainly due to integrated effect of pre and post emergence herbicides resulted in reduced competition among the crop and weed for natural resources like nutrients and moisture. Further, unhindered light penetration increased the fixation of light in photosynthesis thus it expressed by attaining higher plant height (Rajendrakumar et al., 2015). Black polythene mulch controlled the weeds by interrupting the light reaching the weeds and thus reduced the weed intensity and the benefit was witnessed (Nalini, 2007).

Considering the overall economics of the system weed free check treatment showed significantly the highest gross returns (Rs. $81,724 \mathrm{ha}^{-1}$ ) and was closely followed by cotton + sunnhemp (1:1) and in-situ mulching at 45 DAS (Rs. 75,899 $\mathrm{ha}^{-1}$ ) and pendimethalin $38.7 \mathrm{CS} @ 680 \mathrm{~g}$ a.i./ha as PE fb pyrithiobac sodium 10EC $75 \mathrm{~g}$ a.i./ha + quizolofop ethyl $37.5 \mathrm{~g}$ a.i./ha at $25 \mathrm{DAS}$ as POE (Rs.74,284 ha ${ }^{-1}$ ). These treatments were on par with each other but significantly superior over unweeded control (Rs.52, 393 $\mathrm{ha}^{-1}$ ), The highest net returns (Rs. 52,284 ha ${ }^{-1}$ ) was recorded in cotton + sunnhemp (1:1) and in-situ mulching at 45 DAS followed pendimethalin 38.7 CS @ $680 \mathrm{~g}$ a.i./ha as PE fb pyrithiobac sodium 10 EC $75 \mathrm{~g}$ a.i./ha + quizolofop ethyl $37.5 \mathrm{~g}$ a.i./ha at $25 \mathrm{DAS}$ as POE (Rs. 49,613 $\mathrm{ha}^{-1}$ ) and cotton + cowpea (1:1) and in-situ mulching at 45 DAS (Rs.47,283 $\mathrm{ha}^{-1}$ ). Intercropping of cotton + sunnhemp (1:1) and then in-situ mulching at 45 DAS (3.3) recorded significantly higher benefit cost ratio as compared to other treatments. However, it was on par with cotton + cowpea $(1: 1)$ and then in-situ mulching at 45 DAS (3.1) and pendimethalin 38.7 CS @680 g a.i./ha as PE fb pyrithiobac sodium 10EC $75 \mathrm{~g}$ a.i./ha + quizolofop ethyl $37.5 \mathrm{~g}$ a.i./ha at $25 \mathrm{DAS}$ as POE (3.0). The lowest $\mathrm{B}: \mathrm{C}$ was observed in weed free check (Table 4). The higher gross returns was due to higher seed cotton yield and market price, which reflected in less weed population, dry weight of weeds and maximum bolls plant ${ }^{-1}$ (Madhu et al., 2014 and Madavi, 2016). The higher benefit cost ratio was due to reduced cost of cultivation and higher yield which in turn increased the benefit of cultivation. The lower B:C in weed free check and unweeded was mainly due to low gross returns as a result of reduced seed cotton yield and also due to higher cost involved in the production.

In conclusion the eco-friendly treatments viz., cotton + sunnhemp (1:1) and in-situ mulching at 45 DAS, mulching with black polythene, cotton + cowpea (1:1) and in-situ mulching at 45 DAS were at par with weed free check and they might be recommended as these were the best options. These intern would reduces the weed density, increases the growth and yield attribute and increases the yield and net returns and reduces the cost of cultivation and which found to be optimum for improving cotton production for promoting eco-friendly weed management options in cotton cultivation. The other treatments viz., mulching with cotton stalk, paddy straw and spray of leaf extracts were not feasible.

\section{References}

Anonymous, 2018, ICAR-Central Institute for Cotton Research state wise cotton area, production and productivity. www.cicr.org.in/database/dbcapp5.html 
Gomez, K. A. and Gomez, A. A., 1984, Statistical Procedures for Agricultural Research, $\quad 2^{\text {nd }}$ Editn. A wiley Inter-Science Publications, New York (USA).

Jalis, A. and Shah, M.L. 1982. Weed control in cotton resume. The Pak Cottons. 26 (3):141-148.

Lithourgidis, A. S., Dordas, C. A., Damalas, C. A. and Vlachostergios, D. N., 2011, Annual intercrops: an alternative pathway for sustainable agriculture. Australian J. Crop Sci., 5 (4): 396-410.

Lithourgidis, A. S., Vasilakoglou, I. B., Dhima, K. V., Dordas, C. A. and Yiakoulaki, M. D., 2006, Silage yield and quality of common vetch mixtures with oat and triticale in two seeding ratios. Field Crops Res., 99: 106-113.

Madavi, B., Leela Rani, P., Sreenivas, G., Madhavi, A. and Surekha, K.,2017, Impact of high density planting and weed management practices on yield attributes, yield and quality characters of Bt. Cotton. Int. J. Curr. Microbiol. App. Sci., 6(8):194-202.

Madhu, G. Srinivasulu, K., Rani, P. P and Rao, A. S., 2014, Economics of rainfed $B t$ cotton as influenced by sequential application of herbicides. J. Cotton Res. Devpt., 28 (2): 257-259.

Nalini, P., 2007, Poly Mulching - A Case Study to Increase Cotton Productivity. Model Training Course on "Cultivation of long staple cotton (ELS), December15-22 at Central Institute for cotton research, Regional Station, Coimbatore. Pp:183-189.
Patel, J. G., Raj, V. C., Kumar, V., Sutaria, C. M. and Usadadiya, V. P. 2013. Integrated weed management in $\mathrm{Bt}$ cotton. Int. J. Agric. Innov. Res., 2 (2):23-29

Poggio, S. L., 2005, Structure of weed communities occurring in monoculture and intercropping of pea and barley. Agri. Ecosyst. Environ. 109(1-2): 48-58.

Rajendrakumar, 2015, Integrated weed management in Bt Cotton. M. Sc., Thesis, Mahatma Phule Krishi Vidyapeeth, Rahuri, Ahmednagar, Maharashtra, India.

Satishkumar, A., 2016, Studies on the allelopathic effect of intercrops and tree leaf extracts on eco-friendly weed management in irrigated cotton (Gossypium hirsutum 1.). Thesis, Tamil Nadu Agric. Univ., Agric. College and Res. Institute, Madurai, Tamil Nadu, India.

Shivshankar, Channabasavanna, A. S., Ananda, N., Krishnamurthy, D. and Mahadevswamy, 2017, Studies on weed management practices in irrigated $\mathrm{Bt}$ Cotton. J. Farm Sci., 30 (2): 283-284.

Singh, H. P., Batish, D. R. and Kohli, R. K., 2003 Allelopathic interactions and allelochemicals: new possibilities for sustainable weed management. Crit. Rev. Plant Sci. 22: 239-311.

Venugopalan, M. V., Sankaranarayanan, K., Blaise, D., Nalayini, P., Prabhuraj, C. S. and Gangaiah, B., 2009, Bt cotton (Gossypium sp.) in India and its agronomic requirements A review. Indian J. Agronomy. 54 (4): 343-360.

\section{How to cite this article:}

Kamble Anand Shankar, A. S. Channabasavanna, B. G. Koppalkar, M. Y. Ajayakumar, Mahadevswamy and Amaregouda, A. 2020. Evaluation of Non-Chemical Eco-Friendly Weed Management Approaches in HDPS Cotton. Int.J.Curr.Microbiol.App.Sci. 9(11): 3420-3430. doi: https://doi.org/10.20546/ijcmas.2020.911.408 